\title{
Walking-adaptability assessments with the Interactive Walkway: Between-systems agreement and sensitivity to task and subject variations
}

\author{
Daphne J. Geerse ${ }^{\mathrm{a}, \mathrm{b}, *}$, Bert H. Coolen ${ }^{\mathrm{a}}$, Melvyn Roerdink ${ }^{\mathrm{a}}$ \\ ${ }^{a}$ Department of Human Movement Sciences, Faculty of Behavioural and Movement Sciences, Vrije Universiteit Amsterdam, Amsterdam Movement Sciences, \\ The Netherlands \\ ${ }^{\mathrm{b}}$ Department of Neurology, Leiden University Medical Center, Leiden, The Netherlands
}

\section{A R T I C L E I N F O}

\section{Article history:}

Received 1 September 2016

Received in revised form 15 January 2017

Accepted 21 February 2017

\section{Keywords:}

Kinect v2

Walking adaptability

Assessment

Between-systems agreement

Sensitivity to task variation

Sensitivity to subject variation

\begin{abstract}
A B S T R A C T
The ability to adapt walking to environmental circumstances is an important aspect of walking, yet difficult to assess. The Interactive Walkway was developed to assess walking adaptability by augmenting a multi-Kinect-v2 10-m walkway with gait-dependent visual context (stepping targets, obstacles) using real-time processed markerless full-body kinematics. In this study we determined Interactive Walkway's usability for walking-adaptability assessments in terms of between-systems agreement and sensitivity to task and subject variations. Under varying task constraints, 21 healthy subjects performed obstacle-avoidance, sudden-stops-and-starts and goal-directed-stepping tasks. Various continuous walking-adaptability outcome measures were concurrently determined with the Interactive Walkway and a gold-standard motion-registration system: available response time, obstacle-avoidance and sudden-stop margins, step length, stepping accuracy and walking speed. The same holds for dichotomous classifications of success and failure for obstacle-avoidance and sudden-stops tasks and performed short-stride versus long-stride obstacle-avoidance strategies. Continuous walking-adaptability outcome measures generally agreed well between systems (high intraclass correlation coefficients for absolute agreement, low biases and narrow limits of agreement) and were highly sensitive to task and subject variations. Success and failure ratings varied with available response times and obstacle types and agreed between systems for $85-96 \%$ of the trials while obstacle-avoidance strategies were always classified correctly. We conclude that Interactive Walkway walking-adaptability outcome measures are reliable and sensitive to task and subject variations, even in high-functioning subjects. We therefore deem Interactive Walkway walking-adaptability assessments usable for obtaining an objective and more task-specific examination of one's ability to walk, which may be feasible for both high-functioning and fragile populations since walking adaptability can be assessed at various levels of difficulty.
\end{abstract}

(c) 2017 Elsevier B.V. All rights reserved.

\section{Introduction}

An important aspect of walking is one's ability to adapt walking to environmental circumstances [1-3]. Walking adaptability includes the ability to avoid obstacles, make sudden stops and starts and accurately place the feet to environmental context [1]. Most walking-related falls result from inadequate interactions with environmental context, leading to balance loss due to a trip,

\footnotetext{
* Corresponding author at: Vrije Universiteit Amsterdam, Department of Human Movement Sciences, Van der Boechorststraat 7, 1081 BT Amsterdam, The Netherlands.

E-mail address: d.j.geerse@vu.nl (D.J. Geerse).
}

slip or misplaced step [4-6]. Walking adaptability thus seems to be an important determinant of fall risk, yet a comprehensive welltested objective assessment of walking adaptability is lacking [1].

We try to fill this lacuna with the Interactive Walkway (IWW), a 10-m walkway augmented with projected gait-dependent visual context, such as obstacles suddenly appearing at the position one would step next, demanding a step adjustment under time pressure. The basis of the IWW is an integrated multi-Kinect v2 set-up for markerless registration of 3D full-body kinematics during walking [7], which was recently validated over the entire $10-\mathrm{m}$ walkway against a gold standard in 3D measurement accuracy for both kinematics and derived gait parameters $[7,8]$. We have now equipped this set-up with a projector to augment the entire walkway with visual context, such as obstacles, sudden- 
stop-and-start cues and stepping targets, based on real-time processed integrated Kinect data. The so-elicited gait-environment interactions potentially allow for assessing various walkingadaptability aspects (e.g., the ability to avoid obstacles, suddenly stop or start, perform accurate goal-directed steps) as well as subject-specific variations and adaptations affecting walkingadaptability performance (e.g., adopting a slower walking speed to enhance goal-directed stepping accuracy).

The objective of this study is to determine the usability of the IWW for walking-adaptability assessments in a group of healthy adults in terms of between-systems agreement and sensitivity to task and subject variations. Walking-adaptability tasks and associated outcome measures are selected for their proven ability to distinguish between persons who vary in adaptive-walking limitations [2,3,9-12]. To determine the between-systems agreement, IWW-based walking-adaptability outcome measures are compared to those concurrently derived with a gold standard. The sensitivity to task variation is assessed by comparing walkingadaptability performance as a function of context variations, including different obstacle sizes and sequences of stepping targets. Sensitivity to subject variation is explored by quantifying speedperformance trade-offs between self-selected walking speed and adaptive stepping performance (success rates, safety margins). We expect that walking-adaptability outcomes agree well between systems and are sensitive to task and subject variations.

\section{Methods}

\subsection{Subjects}

A heterogeneous group of 21 healthy subjects (mean [range]: age 30 [19-63] years, height $176[158-190] \mathrm{cm}$, weight 70 [53$83] \mathrm{kg}, 11$ males) without severe visual deficits or any medical condition that would affect walking participated. The local ethics committee approved the study. All subjects gave written informed consent prior to participation.

\subsection{Experimental set-up and procedure}

Full-body kinematics for walking over the entire 10-m walkway was obtained with the IWW using four spatially and temporally integrated Kinect v2 sensors (Fig. 1A) and the Optotrak system (Northern Digital Inc., Waterloo, Canada) for 19 matched body points as in [7; see also Supplementary material]. IWW and Optotrak data were sampled at $30 \mathrm{~Hz}$ (using custom-written software utilizing the Kinect-for-Windows Software Development Kit [SDK 2.0]) and $60 \mathrm{~Hz}$ (using First Principles data acquisition software), respectively. The IWW was equipped with a projector (Vivitek D7180HD, ultra-short-throw Full HD projector) to augment the entire $10-\mathrm{m}$ walkway with visual context for three sorts of walking-adaptability tasks: obstacle avoidance, sudden stops-and-starts and goal-directed stepping (Fig. 1).

The obstacle-avoidance task consisted of 25 trials with one or two obstacles (a projected red rectangle) per trial. In total, 40 obstacles were presented, including 20 gait-dependent obstacles (obstacle at predicted foot-placement position appearing two steps ahead; Fig. 1B) and 20 position-dependent obstacles (obstacle at an unpredictable predefined position appearing when a subject's ankle was within $1.5 \mathrm{~m}$ from that obstacle; Fig. 1C). Gait-dependent obstacles were 0.5 (width of the walkway) by $0.3 \mathrm{~m}$. Position-dependent obstacles were larger $(0.5 \mathrm{~m} \times 0.5 \mathrm{~m})$ to increase the need for making step adjustments. Subjects were instructed to avoid suddenly appearing obstacles while walking at self-selected comfortable speeds.

The sudden-stops-and-starts task (Fig. 1D) consisted of 25 trials with in total 40 cues (i.e., one or two sudden-stop-and-start cues per trial) to assess one's ability to suddenly stop and start walking. The cue was a big blue rectangle with a width of $0.5 \mathrm{~m}$ that filled the walkway from an unpredictable predefined position till its end and appeared as soon as a subject's ankle was within $1 \mathrm{~m}$ from this position, triggering the subject to stop walking. After a random period between 5 and $10 \mathrm{~s}$, the rectangle disappeared, triggering the subject to start walking again. Subjects were instructed to walk at self-selected comfortable speeds and to stop behind the cue and to start walking as soon as the cue disappeared.

The goal-directed-stepping task consisted of symmetric-stepping-stones (SSS; Fig. 1E), asymmetric-stepping-stones (ASS; Fig. 1F) and variable-stepping-stones (VSS; Fig. 1G) conditions. Subjects were instructed to step as accurately as possible onto the white shoe-sizematched stepping targets at a self-selected comfortable walking speed. For SSS, seven different imposed step-length trials ranging from 30 to $90 \mathrm{~cm}$ in steps of $10 \mathrm{~cm}$ were performed, all with three repetitions, yielding a total of 21 trials. For ASS, stride length remained $90 \mathrm{~cm}$ while left (L) and right (R) imposed step lengths were varied in separate trials from 15 to $75 \mathrm{~cm}$ in steps of $15 \mathrm{~cm}$ yielding five different imposed stepping asymmetries (L/R: 15/75, 30/60, 45/45, $60 / 30,75 / 15$ ), all with three repetitions, yielding 15 trials. For VSS, imposed step lengths varied within each trial on a step-to-step basis randomly between 30 and $90 \mathrm{~cm}$. Ten different VSS trials were performed, consisting of 21 stepping stones each.

The walking-adaptability tasks were block-randomized and preceded by a familiarization trial. Four ankle-to-shoe calibration trials, in which the subject was standing in two shoe-size-matched

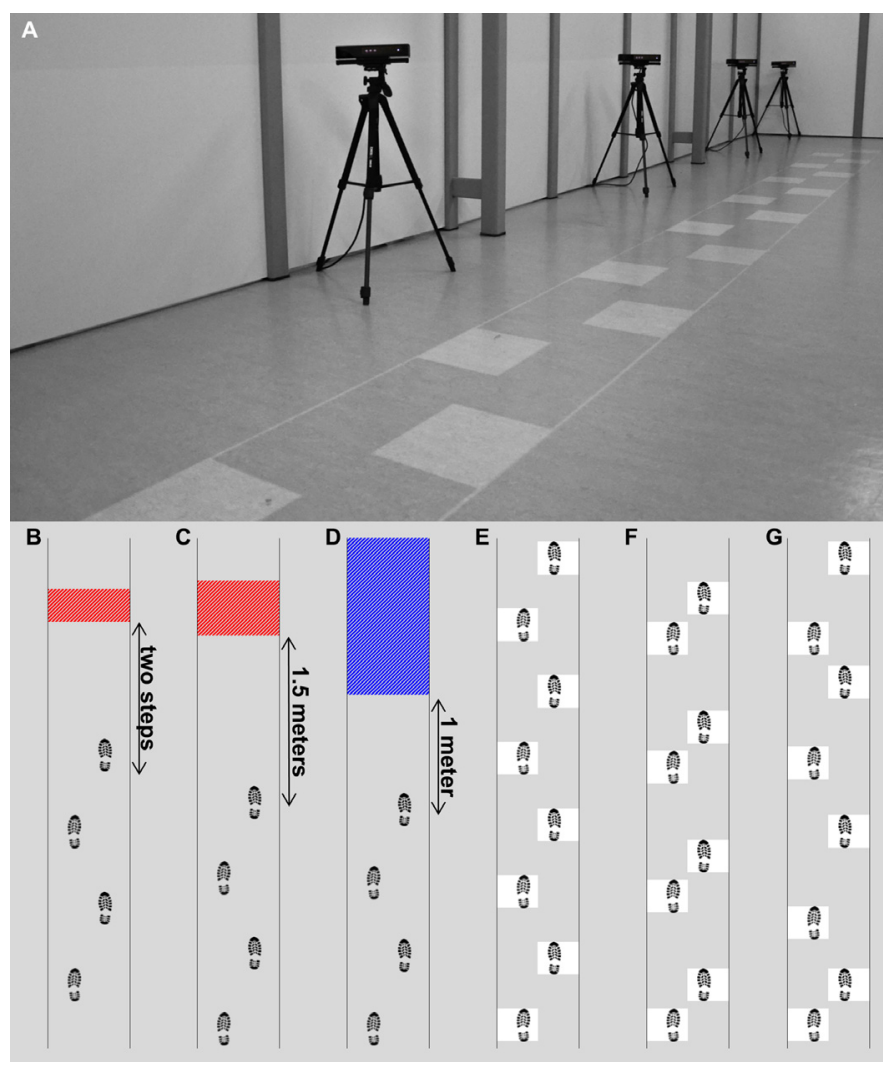

Fig. 1. The set-up of the Interactive Walkway with visual context projected on the walkway (A). The four Kinect v2 sensors were positioned on tripods at a height of $0.75 \mathrm{~m}$ alongside a walkway of 10 by $0.5 \mathrm{~m}$. The sensors were placed frontoparallel (i.e., with an angle of $70^{\circ}$ relative to the walkway direction) with a distance of $0.5 \mathrm{~m}$ from the left border of the walkway. The first sensor was positioned at $4 \mathrm{~m}$ from the start of the walkway and the other sensors were placed at inter-sensor distances of $2.5 \mathrm{~m}$. Schematics of the walking-adaptability tasks: obstacle avoidance with gaitdependent (B) and position-dependent obstacles (C), sudden stops-and-starts (D) and goal-directed stepping with symmetric stepping stones (E), asymmetric stepping stones (F) and variable stepping stones (G). 
Table 1

Calculation methods of continuous walking-adaptability outcome measures.

\begin{tabular}{|c|c|c|c|}
\hline & Outcome measure & Unit & Calculation \\
\hline \multirow[t]{2}{*}{ Obstacle-avoidance task } & Available response time & s & $\begin{array}{l}\text { The distance of the nearest anterior shoe edge to the border of the obstacle at the } \\
\text { moment of its appearance divided by the average walking speed over the second before } \\
\text { its appearance. }\end{array}$ \\
\hline & Obstacle-avoidance margins & $\mathrm{cm}$ & $\begin{array}{l}\text { The distance of the anterior shoe edge (trailing limb) and posterior shoe edge (leading } \\
\text { limb) of the step locations to corresponding obstacle borders during obstacle crossing. } \\
\text { Step locations were determined as the median anterior-posterior position of the ankle } \\
\text { joint during the single-support phase (i.e., between foot off and foot contact of the } \\
\text { contralateral foot) [7]. Estimates of foot contact and foot off were defined as the maxima } \\
\text { and minima of the anterior-posterior time series of the ankles relative to that of the } \\
\text { spine base [7,13]. }\end{array}$ \\
\hline \multirow[t]{2}{*}{ Sudden-stops-and-starts task } & Available response time & s & $\begin{array}{l}\text { The distance of the nearest anterior shoe edge to the border of the sudden-stop cue at } \\
\text { the moment of its appearance divided by the average walking speed over the second } \\
\text { before its appearance. }\end{array}$ \\
\hline & Sudden-stop margin & $\mathrm{cm}$ & $\begin{array}{l}\text { The minimum distance of the anterior shoe edge to the corresponding sudden-stop cue } \\
\text { border during the period in which the cue was visible. }\end{array}$ \\
\hline \multirow[t]{3}{*}{ Goal-directed-stepping task } & Step length & $\mathrm{cm}$ & $\begin{array}{l}\text { The median of the differences in the anterior-posterior direction of consecutive step } \\
\text { locations. Step locations were determined as the median anterior-posterior position of } \\
\text { the ankle joint during the single-support phase (i.e., between foot off and foot contact of } \\
\text { the contralateral foot) [7]. Estimates of foot contact and foot off were defined as the } \\
\text { maxima and minima of the anterior-posterior time series of the ankles relative to that } \\
\text { of the spine base [7,13]. }\end{array}$ \\
\hline & Stepping accuracy & $\mathrm{cm}$ & $\begin{array}{l}\text { The standard deviation over the signed deviations between the center of the foot and } \\
\text { the center of the target at step locations, as defined in step length. Stepping accuracy } \\
\text { was determined over step locations that were identified for both systems to ensure a } \\
\text { fair comparison. The center of the foot was determined using the average distance } \\
\text { between the ankle and the middle of the shoe-size-matched targets of the calibration } \\
\text { trials. }\end{array}$ \\
\hline & Walking speed & $\mathrm{cm} / \mathrm{s}$ & $\begin{array}{l}\text { The distance traveled between the start and the } 10-\mathrm{m} \text { line of the walkway divided by } \\
\text { the duration, using the data of the spine shoulder. }\end{array}$ \\
\hline
\end{tabular}

targets at different positions on the walkway, were also included to determine the average distance between shoe edges and the ankle for both systems. This calibration was needed to determine several walking-adaptability outcome measures (see below).

\subsection{Data pre-processing and analysis}

Data pre-processing followed established procedures [7]; details about the procedure and pre-processed data are presented as Supplementary material. Due to excessive missing data, 62 out of 2016 trials were excluded from further analysis, mainly for the gold-standard motion-registration system (i.e., marker occlusion and/or orientation issues) and concerning one subject.

The continuous walking-adaptability outcome measures were available response time (ART) and margins of the trailing and leading limb during obstacle crossing for the obstacle-avoidance task, ART and margin to the stop cue for the sudden-stops-andstarts task, step length, stepping accuracy and walking speed for SSS and VSS, and left and right step lengths, stepping accuracy and walking speed for ASS. These continuous outcome measures were calculated from specific body points' time series, estimates of foot contact and foot off and step locations, as detailed in Table 1, for both measurement systems alike in an aligned coordinate system, including the coordinates of obstacles, sudden-stop cues and targets. For all continuous outcome measures, statistical analyses were performed over averages over trials. For dichotomous outcome measures, step locations were extrapolated to the actual shoe dimensions based on the ankle-to-shoe calibration to determine whether or not obstacle-avoidance and sudden-stop trials were successfully performed, from which success rates were deduced. Successful gait-dependent obstacle-avoidance maneuvers were classified as short-stride or long-stride strategies [14].

\subsection{Statistical analysis}

Between-systems agreement was determined for continuous outcome measures using intraclass correlation coefficients for absolute agreement $\left(\operatorname{ICC}_{(\mathrm{A}, 1)}\right.$; [15]), with values above 0.60 and 0.75 representing good and excellent agreement, respectively [16]. This analysis of between-systems agreement was complemented by mean differences and precision values obtained with a Bland-Altman analysis (i.e., the bias and the limits of agreement, respectively [17]). For dichotomous outcome measures we report the percentage of non-matched ratings.

Sensitivity to task variation was examined using repeatedmeasures ANOVAs on continuous outcome measures of obstacleavoidance and goal-directed-stepping tasks. For ART and obstacleavoidance margins, a System (IWW, Optotrak) by Obstacle (gaitdependent, position-dependent) by Limb (trailing, leading) repeated-measures ANOVA was conducted. For step length, stepping accuracy and walking speed of SSS, a System by Imposed step length $(30,40, \ldots, 90)$ repeated-measures ANOVA was conducted. For left and right step lengths, stepping accuracy and walking speed of ASS, a System by Imposed step-length asymmetry (L/R: $15 / 75,30 / 60,45 / 45,60 / 30,75 / 15)$ repeated-measures ANOVA was conducted. For step length, stepping accuracy and walking speed of VSS, a System by Trial repeated-measures ANOVA was conducted. For the average stepping accuracy of the three goal-directedstepping conditions, a System by Condition (SSS, ASS, VSS) repeated-measures ANOVA was conducted. One subject was excluded from the analyses of the goal-directed-stepping tasks due to multiple trials with excessive missing values. The assumption of sphericity was checked according to Girden [18]. If Greenhouse-Geisser's epsilon exceeded 0.75, the HuynhFeldt correction was applied; otherwise the Greenhouse-Geisser correction was used. Main effects were examined with a LSD post hoc test for factors with three levels and contrast analyses for factors with more than three levels. Paired-samples $t$-tests were used for significant interactions. Effect sizes were quantified with $\eta_{p}^{2}$.

Sensitivity to subject variation was examined by exploring speed-performance trade-offs. We determined Pearson's correlations between self-selected walking speed and stepping accuracy for all goal-directed-stepping tasks and between the 
Table 2

Agreement statistics for continuous outcome measures of obstacle-avoidance, sudden-stops-and-starts and goal-directed-stepping (SSS, ASS and VSS) tasks.

\begin{tabular}{|c|c|c|c|c|c|c|}
\hline & & & Interactive Walkway mean \pm SD & Optotrak system mean $\pm S D$ & Bias $[95 \%$ LoA] & $\operatorname{ICC}_{(A, 1)}$ \\
\hline \multicolumn{7}{|l|}{ Obstacle-avoidance task } \\
\hline \multirow[t]{2}{*}{ ART (s) } & Gait-dependent & & $0.792 \pm 0.050$ & $0.777 \pm 0.049$ & $-0.015^{*}\left[\begin{array}{ll}-0.032 & 0.002\end{array}\right]$ & 0.945 \\
\hline & Position-dependent & & $0.834 \pm 0.075$ & $0.834 \pm 0.076$ & $0.000\left[\begin{array}{lll}-0.023 & 0.024\end{array}\right]$ & 0.988 \\
\hline \multirow[t]{4}{*}{ Margins $(\mathrm{cm})$} & Gait-dependent & Trailing limb & $27.68 \pm 5.53$ & $27.65 \pm 5.06$ & $-0.03\left[\begin{array}{lll}-2.17 & 2.12\end{array}\right]$ & 0.980 \\
\hline & & Leading limb & $11.68 \pm 5.45$ & $12.78 \pm 5.26$ & $1.11^{*}[-1.353 .56]$ & 0.954 \\
\hline & Position-dependent & Trailing limb & $11.27 \pm 3.08$ & $11.54 \pm 2.90$ & $0.26\left[\begin{array}{ll}-2.18 & 2.71]\end{array}\right]$ & 0.913 \\
\hline & & Leading limb & $8.97 \pm 4.91$ & $9.82 \pm 4.87$ & $0.85^{*}[-1.393 .09]$ & 0.960 \\
\hline \multicolumn{7}{|c|}{$\overline{\text { Sudden-stops-and-starts task }}$} \\
\hline ART $(\mathrm{s})$ & & & $0.497 \pm 0.067$ & $0.490 \pm 0.070$ & $-0.007^{*}\left[\begin{array}{lll}-0.035 & 0.021]\end{array}\right.$ & 0.997 \\
\hline Margins (cm) & & & $8.32 \pm 7.29$ & $8.35 \pm 6.70$ & $0.30[-6.967 .02]$ & 0.876 \\
\hline \multicolumn{7}{|l|}{ SSS } \\
\hline \multirow[t]{7}{*}{ Step length $(\mathrm{cm})$} & 30 & & $29.95 \pm 0.14$ & $29.97 \pm 0.32$ & $0.02\left[\begin{array}{ll}-0.55 & 0.58\end{array}\right]$ & 0.339 \\
\hline & 40 & & $39.96 \pm 0.18$ & $40.00 \pm 0.28$ & $0.04\left[\begin{array}{ll}-0.61 & 0.68\end{array}\right]$ & 0.034 \\
\hline & 50 & & $50.06 \pm 0.29$ & $50.02 \pm 0.35$ & $-0.04\left[\begin{array}{lll}-1.04 & 0.96\end{array}\right]$ & -0.276 \\
\hline & 60 & & $60.02 \pm 0.38$ & $59.89 \pm 0.48$ & $-0.13\left[\begin{array}{ll}-1.21 & 0.95\end{array}\right]$ & 0.189 \\
\hline & 70 & & $69.99 \pm 0.25$ & $69.91 \pm 0.57$ & $-0.07\left[\begin{array}{ll}-1.05 & 0.90\end{array}\right]$ & 0.376 \\
\hline & 80 & & $79.89 \pm 0.28$ & $79.76 \pm 0.48$ & $-0.13\left[\begin{array}{lll}-1.10 & 0.84\end{array}\right]$ & 0.210 \\
\hline & 90 & & $89.84 \pm 0.37$ & $89.81 \pm 0.33$ & $-0.03\left[\begin{array}{ll}-0.82 & 0.76\end{array}\right]$ & 0.367 \\
\hline \multirow[t]{7}{*}{ Stepping accuracy $(\mathrm{cm})$} & 30 & & $1.77 \pm 0.41$ & $1.87 \pm 0.38$ & $0.10\left[\begin{array}{lll}-0.55 & 0.75\end{array}\right]$ & 0.635 \\
\hline & 40 & & $1.80 \pm 0.37$ & $1.93 \pm 0.45$ & $0.13\left[\begin{array}{lll}-0.66 & 0.92\end{array}\right]$ & 0.503 \\
\hline & 50 & & $1.81 \pm 0.37$ & $2.00 \pm 0.47$ & $0.20^{*}[-0.490 .88]$ & 0.609 \\
\hline & 60 & & $1.91 \pm 0.46$ & $1.91 \pm 0.52$ & $0.00[-0.770 .78]$ & 0.686 \\
\hline & 70 & & $1.91 \pm 0.41$ & $1.99 \pm 0.49$ & $0.08\left[\begin{array}{lll}-0.64 & 0.80\end{array}\right]$ & 0.675 \\
\hline & 80 & & $1.88 \pm 0.54$ & $2.02 \pm 0.53$ & $0.15[-0.891 .19]$ & 0.498 \\
\hline & 90 & & $2.02 \pm 0.55$ & $2.12 \pm 0.56$ & $0.10\left[\begin{array}{ll}-0.59 & 0.78\end{array}\right]$ & 0.798 \\
\hline \multirow[t]{7}{*}{ Walking speed $(\mathrm{cm} / \mathrm{s})$} & 30 & & $73.23 \pm 12.95$ & $72.89 \pm 12.66$ & $-0.34^{*}\left[\begin{array}{ll}-1.03 & 0.35]\end{array}\right.$ & 0.999 \\
\hline & 40 & & $86.93 \pm 13.42$ & $86.37 \pm 13.04$ & $-0.57^{*}\left[\begin{array}{ll}-1.48 & 0.35\end{array}\right]$ & 0.999 \\
\hline & 50 & & $101.14 \pm 14.11$ & $100.42 \pm 13.73$ & $-0.72^{*}\left[\begin{array}{lll}-1.67 & 0.23\end{array}\right]$ & 0.998 \\
\hline & 60 & & $112.28 \pm 13.83$ & $111.19 \pm 13.28$ & $-1.09^{*}\left[\begin{array}{ll}-2.57 & 0.39\end{array}\right]$ & 0.995 \\
\hline & 70 & & $124.40 \pm 13.38$ & $123.24 \pm 12.89$ & $-1.16^{*}\left[\begin{array}{lll}-2.59 & 0.26\end{array}\right]$ & 0.995 \\
\hline & 80 & & $136.70 \pm 12.49$ & $134.97 \pm 12.07$ & $-1.73^{*}[-3.00-0.46]$ & 0.989 \\
\hline & 90 & & $145.07 \pm 12.07$ & $143.43 \pm 11.67$ & $-1.64^{*}[-3.10-0.19]$ & 0.989 \\
\hline \multicolumn{7}{|l|}{$\overline{\mathrm{ASS}}$} \\
\hline \multirow[t]{5}{*}{ Step length left $(\mathrm{cm})$} & $15 / 75$ & & $21.38 \pm 3.66$ & $19.75 \pm 3.92$ & $-1.63^{*}[-4.301 .03]$ & 0.859 \\
\hline & $30 / 60$ & & $34.23 \pm 2.39$ & $33.55 \pm 2.71$ & $-0.68\left[\begin{array}{ll}-3.65 & 2.29\end{array}\right]$ & 0.803 \\
\hline & $45 / 45$ & & $44.72 \pm 1.17$ & $44.50 \pm 1.76$ & $-0.22\left[\begin{array}{ll}-3.03 & 2.59\end{array}\right]$ & 0.546 \\
\hline & $60 / 30$ & & $55.44 \pm 2.35$ & $56.34 \pm 2.82$ & $0.90^{*}\left[\begin{array}{ll}-2.03 & 3.83\end{array}\right]$ & 0.793 \\
\hline & $75 / 15$ & & $67.44 \pm 2.96$ & $69.88 \pm 3.58$ & $2.45^{*}[-0.965 .86]$ & 0.677 \\
\hline \multirow[t]{5}{*}{ Step length right $(\mathrm{cm})$} & $15 / 75$ & & $68.57 \pm 3.84$ & $70.16 \pm 3.96$ & $1.60^{*}[-1.414 .61]$ & 0.854 \\
\hline & $30 / 60$ & & $55.76 \pm 2.58$ & $56.45 \pm 2.84$ & $0.69[-2.483 .86]$ & 0.803 \\
\hline & $45 / 45$ & & $45.37 \pm 1.24$ & $45.39 \pm 1.87$ & $0.01\left[\begin{array}{ll}-2.85 & 2.88\end{array}\right]$ & 0.588 \\
\hline & $60 / 30$ & & $34.62 \pm 2.20$ & $33.63 \pm 2.66$ & $-0.99^{*}\left[\begin{array}{lll}-3.74 & 1.76\end{array}\right]$ & 0.777 \\
\hline & $75 / 15$ & & $22.80 \pm 2.89$ & $19.96 \pm 3.56$ & $-2.83^{*}\left[\begin{array}{lll}-6.37 & 0.71]\end{array}\right.$ & 0.615 \\
\hline \multirow[t]{5}{*}{ Stepping accuracy $(\mathrm{cm})$} & $15 / 75$ & & $3.87 \pm 1.77$ & $3.37 \pm 1.58$ & $-0.50^{*}\left[\begin{array}{ll}-1.75 & 0.75]\end{array}\right.$ & 0.891 \\
\hline & $30 / 60$ & & $2.87 \pm 1.13$ & $2.65 \pm 1.08$ & $-0.21[-1.541 .11]$ & 0.806 \\
\hline & $45 / 45$ & & $1.73 \pm 0.38$ & $1.88 \pm 0.46$ & $0.14\left[\begin{array}{lll}-0.59 & 0.88\end{array}\right]$ & 0.584 \\
\hline & $60 / 30$ & & $3.02 \pm 1.03$ & $2.79 \pm 1.03$ & $-0.23\left[\begin{array}{lll}-1.20 & 0.74\end{array}\right]$ & 0.869 \\
\hline & $75 / 15$ & & $4.36 \pm 1.36$ & $3.34 \pm 1.49$ & $-1.02^{*}\left[\begin{array}{ll}-2.35 & 0.31\end{array}\right]$ & 0.709 \\
\hline \multirow[t]{5}{*}{ Walking speed $(\mathrm{cm} / \mathrm{s})$} & $15 / 75$ & & $90.87 \pm 12.05$ & $90.33 \pm 11.81$ & $-0.54^{*}\left[\begin{array}{lll}-1.34 & 0.25]\end{array}\right.$ & 0.998 \\
\hline & $30 / 60$ & & $92.01 \pm 13.61$ & $91.46 \pm 13.35$ & $-0.55^{*}[-1.430 .34]$ & 0.999 \\
\hline & $45 / 45$ & & $91.73 \pm 14.14$ & $91.20 \pm 13.96$ & $-0.53^{*}\left[\begin{array}{lll}-1.34 & 0.28\end{array}\right]$ & 0.999 \\
\hline & $60 / 30$ & & $89.23 \pm 14.18$ & $88.75 \pm 13.92$ & $-0.47^{*}\left[\begin{array}{lll}-1.24 & 0.29\end{array}\right]$ & 0.999 \\
\hline & $75 / 15$ & & $87.84 \pm 13.51$ & $87.31 \pm 13.25$ & $-0.53^{*}\left[\begin{array}{ll}-1.33 & 0.26\end{array}\right]$ & 0.999 \\
\hline \multicolumn{7}{|l|}{$\overline{\text { VSS }}$} \\
\hline Step length $(\mathrm{cm})$ & & & $45.54 \pm 0.82$ & $45.49 \pm 0.85$ & $-0.05\left[\begin{array}{lll}-0.96 & 0.86\end{array}\right]$ & 0.852 \\
\hline Stepping accuracy $(\mathrm{cm})$ & & & $2.60 \pm 0.68$ & $2.53 \pm 0.65$ & $-0.08\left[\begin{array}{lll}-0.59 & 0.44]\end{array}\right.$ & 0.920 \\
\hline Walking speed $(\mathrm{cm} / \mathrm{s})$ & & & $97.89 \pm 13.88$ & $97.25 \pm 13.56$ & $-0.64^{*}\left[\begin{array}{ll}-1.51 & 0.23\end{array}\right]$ & 0.998 \\
\hline
\end{tabular}


agreement $\left.\left[\operatorname{ICC}_{(\mathrm{A}, 1)}\right]\right)$ for the continuous outcome measures of the obstacle-avoidance, sudden-stops-and-starts and goal-directed-stepping tasks.

ART = available response time, SSS = symmetric stepping stones, ASS = asymmetric stepping stones, VSS=variable stepping stones.

Significant between-systems difference $(p<0.05)$.

speed-dependent ART and margins for obstacle-avoidance and sudden-stop tasks (i.e., significant positive correlations signal speed-performance trade-offs). We also assessed the influence of obstacle-avoidance and sudden-stop ratings on ART using a System by Rating (success, failure) repeated-measures ANOVA. In addition, obstacle-avoidance success rates were compared with a System by Obstacle repeated-measures ANOVA.

\section{Results}

\subsection{Between-systems agreement}

Excellent between-systems agreement was observed for ART and margins for obstacle-avoidance and sudden-stops-and-starts tasks, walking speed for all goal-directed-stepping conditions (SSS, 
ASS and VSS) and step length and stepping accuracy of VSS, supported by very high $\operatorname{ICC}_{(\mathrm{A}, 1)}$ values, small biases and narrow limits of agreement (Table 2 ). The between-systems agreement for stepping accuracy of SSS and step lengths and stepping accuracy for ASS was overall good to excellent (Table 2). Between-systems statistics were ambiguous for step length of SSS (low $\operatorname{ICC}_{(A, 1)}$ values, negligible biases and very narrow limits of agreement; Table 2). Significant between-system biases, indicated in Table 2, all corresponded to significant System effects of associated outcome measures in the ANOVAs for the analysis of sensitivity to task and subject variations.

Success rates of gait-dependent and position-dependent obstacles were (mean \pm SD) $94.7 \pm 12.8 \%$ and $92.1 \pm 15.6 \%$ for the IWW and $96.8 \pm 6.5 \%$ and $93.2 \pm 12.1 \%$ for the gold standard, respectively. The percentage of non-matched ratings was $3.7 \%$ for gait-dependent obstacles (3.0\% false negatives) and $5.1 \%$ for positiondependent obstacles (3.1\% false negatives). Given the uneven distribution of ratings over categories ( $\sim 95 \%$ success vs. $\sim 5 \%$ failure), we also determined the percentages of specific agreement [19] for obstacle-avoidance successes (97.7\%) and failures (61.5\%), suggesting that the agreement for failures was considerably lower. The systems matched perfectly for classified avoidance strategies ( $0 \%$ nonmatched ratings), with an overall preference for the long-stride strategy in avoiding gait-dependent obstacles ( $80.5 \pm 15.3 \%)$. Success rates for sudden stops were $58.1 \pm 23.5 \%$ for the IWW and $49.5 \pm 22.0 \%$ for the gold standard, with $14.8 \%$ between-systems dis-matches ( $11.7 \%$ false positives).

\subsection{Sensitivity to task variation}

A significant obstacle $\left(F(1,20)=7.98, p=0.010, \eta_{p}^{2}=0.285\right)$ effect was found for ART, with longer ARTs for position-dependent obstacles $(0.834 \pm 0.016 \mathrm{~s})$ than for gait-dependent obstacles $(0.784 \pm 0.011 \mathrm{~s})$. Significant obstacle $(F(1,20)=508.73, p<0.001$, $\left.\eta_{p}^{2}=0.962\right)$ and $\operatorname{limb}\left(F(1,20)=29.40, p<0.001, \eta_{p}^{2}=0.595\right)$ effects were found for obstacle-avoidance margins, as well as a significant obstacle $\times$ limb interaction $\left(F(1,20)=99.95, p<0.001, \eta_{p}^{2}=0.833\right)$. While margins were overall greater for gait-dependent obstacles and for the trailing limb, the interaction revealed that the difference between trailing and leading limbs was only evident for gaitdependent obstacles $(27.7 \pm 5.3 \mathrm{~cm}$ vs. $12.2 \pm 5.3 \mathrm{~cm})$ and not for position-dependent obstacles $(11.4 \pm 2.9 \mathrm{~cm}$ vs. $9.4 \pm 4.9 \mathrm{~cm})$.

Subjects were well able to adjust their foot placement to the presented goal-directed-stepping targets (Table 2 and Fig. 2). This was confirmed by very strong effects of imposed step lengths on performed step lengths for SSS $(F(4.2,79.0)=162,327.08, p<0.001$, $\eta_{p}^{2}=1.000$; Fig. 2A) and ASS (left: $F(1.2,22.6)=936.64, p<0.001$, $\eta_{p}^{2}=0.980$; right: $F(1.2,22.7)=913.62, \quad p<0.001, \quad \eta_{p}^{2}=0.980$; Fig. 2B). Stepping accuracy varied significantly with imposed step-length asymmetry $\quad(F(2.4,45.7)=20.63, \quad p<0.001$, $\left.\eta_{p}^{2}=0.521\right)$, with significant quadratic $(F(1,19)=53.99, p<0.001$, $\left.\eta_{p}^{2}=0.740\right)$ and fourth-order $(F(1,19)=18.83, \quad p<0.001$, $\eta_{p}^{2}=0.498$ ) contrasts (Fig. 2E); no significant main or interaction effects were found on stepping accuracy for SSS (Fig. 2D) or VSS (Fig. 2F). Walking speed varied with step-length manipulations for SSS $\left(F(2.7,50.6)=607.50, p<0.001, \eta_{p}^{2}=0.970\right.$; with significant linear $\left[F(1,19)=1189.66, p<0.001, \eta_{p}^{2}=0.984\right]$ and quadratic $\left[F(1,19)=9.29, p=0.007, \eta_{p}^{2}=0.328\right]$ contrasts; Fig. $\left.2 \mathrm{G}\right)$ and ASS $\left(F(2.7,50.6)=4.72, p=0.007, \eta_{p}^{2}=0.199\right.$; with a significant linear contrast $\left[F(1,19)=13.67, p=0.002, \eta_{p}^{2}=0.418\right]$; Fig. $\left.2 \mathrm{H}\right)$. Average stepping accuracy varied significantly over goal-directed-stepping conditions $\left(F(1.5,28.3)=36.80, p<0.001, \eta_{p}^{2}=0.659\right)$; stepping accuracy improved from ASS $(2.99 \pm 0.21 \mathrm{~cm})$ to VSS $(2.57 \pm 0.15 \mathrm{~cm})$ to SSS $(1.93 \pm 0.08 \mathrm{~cm})$, with significant differences between all conditions.

\subsection{Sensitivity to subject variation}

Self-selected walking speed affects the available response time for obstacle-avoidance and sudden-stop tasks on the IWW, and thereby the difficulty of these walking-adaptability tasks. For sudden stops the overall success rate was $53.8 \pm 22.4 \%$, with a clear influence of rating on ART $\left(F(1,20)=172.88, p<0.001, \eta_{p}^{2}=0.896\right)$; ARTs were longer for successful stops $(0.536 \pm 0.012 \mathrm{~s})$ than for failed stops $(0.416 \pm 0.012 \mathrm{~s})$. In Fig. 3 sudden-stop success and failure rates are depicted as a function of ART, showing a steady increase in stopping successes (and hence a decrease in stopping failures) with longer ARTs. A speed-performance trade-off was also found on margins to the stopping cue, with longer ARTs being associated with larger margins, for both systems alike (IWW: $r(20)=0.597, p=0.004$; gold standard: $r(20)=0.698, p<0.001)$.

The influence of obstacle-avoidance ratings on ART could not be determined because of a ceiling effect; overall success rate was $94.2 \pm 11.3 \%$, with slightly higher success rates for gait-dependent obstacles $(95.8 \pm 2.1 \%)$ than for position-dependent obstacles $(92.6 \pm 2.9 \%$; main obstacle effect, $F(1,20)=7.05, \quad p=0.015$, $\left.\eta_{p}^{2}=0.261\right)$. Obstacle-avoidance margins were not associated with ART (i.e., no speed-performance trade-off; $r(20)=\left[\begin{array}{ll}-0.1150 .211 & \text {, }\end{array}\right.$ $p>0.359)$

Clear speed-performance trade-offs were observed for goaldirected stepping, with faster walking speeds being associated with poorer stepping accuracy, as evidenced by significant positive correlations between self-selected walking speed and stepping accuracy for SSS, ASS and VSS, for both systems alike (IWW: $r(20)=0.722, p<0.001, r(20)=0.715, p<0.001$ and $r(20)=0.637$, $p<0.001$, respectively; gold standard: $r(20)=0.523, p=0.018$, $r(20)=0.668, p=0.001$ and $r(20)=0.569, p<0.001$, respectively .

\section{Discussion}

We determined the usability of IWW walking-adaptability assessments in a group of healthy adults in terms of betweensystems agreement and sensitivity to task and subject variations. We expected that walking-adaptability outcome measures agreed well between systems and were sensitive to task and subject variations. The results were in line with our expectations, which led us to conclude that the IWW is usable for walking-adaptability assessments.

First, the between-systems agreement for continuous walkingadaptability outcomes proved to be good to excellent, with high ICC values, small biases and narrow limits of agreement (Table 2). For the SSS conditions of goal-directed stepping, however, ICC values for step length were considerably lower, suggesting a poor between-systems agreement, which stood in stark contrast with excellent Bland-Altman agreement statistics (negligible biases and narrow limits of agreement; Table 2). This discrepancy was likely due to a lack of subject heterogeneity in step lengths since these were experimentally imposed with stepping targets, yielding minimal between-subject variance (see also Fig. 2A) and hence arbitrarily low ICC values [20]. This discrepancy illustrates the importance of a complementary set of agreement statistics instead of relying solely on ICC as the measure for between-systems agreement [20]. The between-systems agreement for dichotomous walking-adaptability outcomes varied, ranging from 100\% overall agreement for obstacle-avoidance strategies to $85.2 \%$ for successes and failures in sudden stops. The specific agreement for obstacleavoidance failures was lower ( $60 \%)$, yet based on a limited number of observations. Future research may exploit IWW's possibility to vary task difficulty to achieve a similar distribution of obstacle-avoidance successes and failures to properly quantify their between-systems agreement. 
A
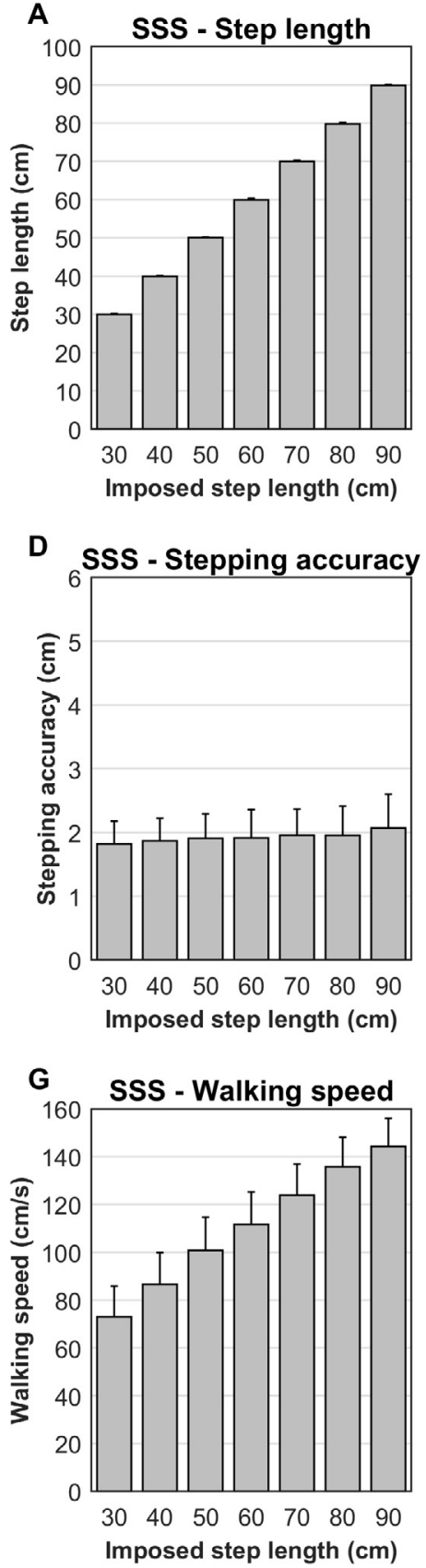

B



E



Imposed step-length asymmetry $(\mathrm{cm})$

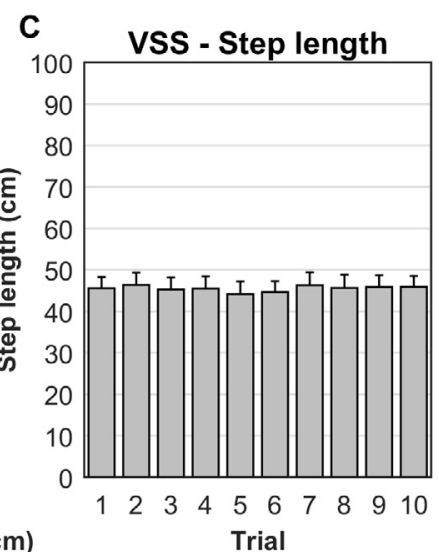

F VSS-Stepping accuracy

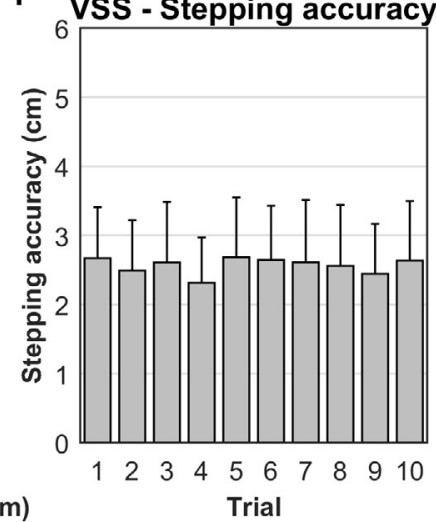

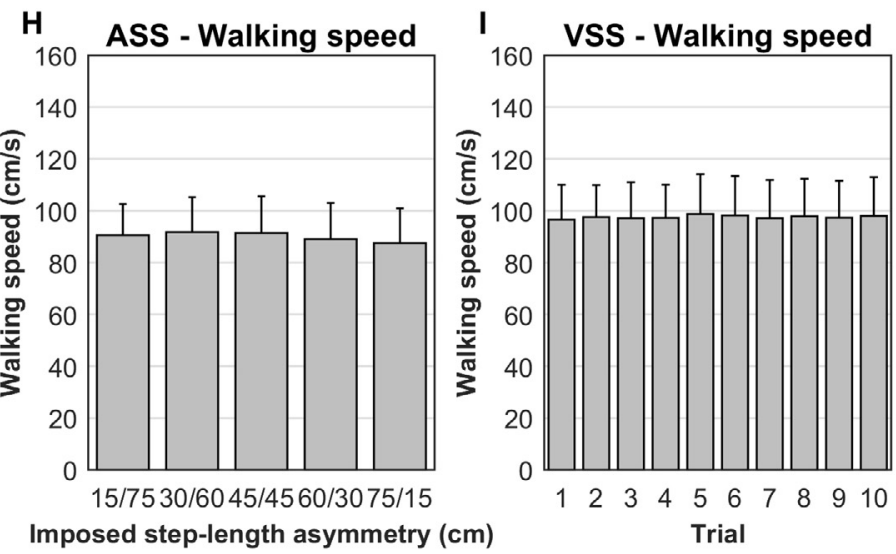

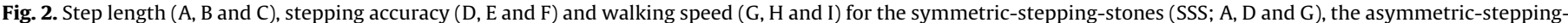
stones (ASS; B, E and H) and the variable-stepping-stones (VSS; C, F and I) of the goal-directed-stepping task.

Second, continuous walking-adaptability outcomes were sensitive to task and subject variations. With goal-directed stepping, task variations led to different step lengths, stepping accuracies and walking speeds (Fig. 2) while ARTs and margins of the trailing limb varied with obstacle type. This testifies to the power of projected visual context in modifying gait and in eliciting (sudden) step adjustments, in line with previous studies exploring the same concept during treadmill walking [3,21-23], as well as to the sensitivity of continuous walking-adaptability outcomes. Success rates differed between obstacle types, although differences were very small in the vicinity of a ceiling effect. Future studies may increase obstacle-avoidance difficulty with the IWW by reducing ART, projecting larger obstacles, and/or adding attention-demanding secondary tasks [24]. Varying task difficulty with ART manipulations seems particularly effective, since in the present study ART had a prominent effect on sudden-stop success rates (Fig. 3) and in other studies on obstacle-avoidance success rates $[12,25]$. Sensitivity to subject variation was further demonstrated by speed-performance trade-offs in goal-directed stepping (subjects who walked faster stepped less accurately onto targets) and sudden stops (subjects with shorter ARTs had smaller margins to the stop cue). Revealing such context-dependent interactions by objectively quantifying a complementary set of outcome measures can be considered one of the strengths of the IWW, which may prove useful in identifying fallers [26] and designing tailored interventions to reduce fall risk [1]. 




Fig. 3. Sudden-stop success and failure rates for different available response times.

Taken together, our results confirmed that IWW walkingadaptability outcome measures are reliable (albeit that obstacleavoidance failure rates have to be considered with caution) and sensitive to task and subject variations, even in high-functioning subjects. Sensitivity to task and subject variations is important for walking-adaptability assessments in relatively high-functioning groups (such as community-dwelling older adults), where ceiling effects are a common concern in fall-risk assessments [27]. The same holds for floor effects in relatively fragile groups (such as fallprone populations). The IWW potentially allows for walkingadaptability assessments that are feasible for both high-functioning and fragile populations since task difficulty can be varied. IWW assessments are also relatively safe (e.g., visual instead of physical obstacles), unobtrusive (markerless data) and hence time-efficient and patient-friendly. The premise is that persons at risk of falling during walking may be better identified with task-specific assessments attuned to common causes and circumstances of falls [4-6], such as IWW walking-adaptability tasks. Future studies are warranted to determine which walking-adaptability tasks and associated outcomes are good indicators of safe walking and accurate predictors of falls during walking.

\section{Acknowledgements}

We are grateful to Microsoft for accepting MR and $\mathrm{HC}$ as members of the Kinect for Windows developers program, allowing us to work with the Kinect v2 sensor and SDK before its commercial release. We would like to acknowledge Aukje de Vrijer from NDI for her invaluable help with setting up the Optotrak system and introducing us to the First Principles software. This work is part of the research program Technology in Motion (TIM [628.004.001]), which is financed by the Netherlands Organisation for Scientific Research (NWO). The funder had no role in the study design, data collection and analysis, interpretation of data, decision to publish, or writing of the manuscript.

\section{Conflict of interest statement}

The authors declare that there is no conflict of interest.

\section{Appendix A. Supplementary data}

Supplementary data associated with this article can be found, in the online version, at http://dx.doi.org/10.1016/j.gaitpost.2017. 02.021 .

\section{References}

[1] C.K. Balasubramanian, D.J. Clark, E.J. Fox, Walking adaptability after a stroke and its assessment in clinical settings, Stroke Res. Treat. (2014) 591013.

[2] M.J. Caetano, S.R. Lord, D. Schoene, P.H. Pelicioni, D.L. Sturnieks, J.C. Menant, Agerelated changes in gait adaptability in response to unpredictable obstacles and stepping targets, Gait Posture 46 (2016) 35-41.

[3] H. Houdijk, M.W. van Ooijen, J.J. Kraal, H.O. Wiggerts, W. Polomski, T.W. Janssen, M. Roerdink, Assessing gait adaptability in people with a unilateral amputation on an instrumented treadmill with a projected visual context, Phys. Ther. 92 (11) (2012) 1452-1460.

[4] W.P. Berg, H.M. Alessio, E.M. Mills, C. Tong, Circumstances and consequences of falls in independent community-dwelling older adults, Age Ageing 26 (4) (1997) 261-268.

[5] A. Forster, J. Young, Incidence and consequences of falls due to stroke: a systematic inquiry, BMJ 311 (6997) (1995) 83-86

[6] D. Hyndman, A. Ashburn, E. Stack, Fall events among people with stroke living in the community: circumstances of falls and characteristics of fallers, Arch. Phys. Med. Rehabil. 83 (2) (2002) 165-170.

[7] D.J. Geerse, B.H. Coolen, M. Roerdink, Kinematic validation of a multi-kinect v2 instrumented 10-meter walkway for quantitative gait assessments, PLOS ONE 10 (10) (2015), e0139913

[8] S. Springer, G. Yogev Seligmann, Validity of the kinect for gait assessment: a focused review, Sensors (Basel) 16 (2) (2016) 194.

[9] K.L. Hollands, T.A. Pelton, S. van der Veen, S. Alharbi, M.A. Hollands, A novel and simple test of gait adaptability predicts gold standard measures of functional mobility in stroke survivors, Gait Posture 43 (2016) 170-175.

[10] U. Lindemann, J. Klenk, C. Becker, R. Moe-Nilssen, Assessment of adaptive walking performance, Med. Eng. Phys. 35 (2) (2013) 217-220.

[11] M. Mazaheri, M. Roerdink, R.J. Bood, J. Duysens, P.J. Beek, C.L. Peper, Attentional costs of visually guided walking: effects of age, executive function and steppingtask demands, Gait Posture 40 (1) (2014) 182-186.

[12] R. Van Swigchem, H. van Duijnhoven, J. den Boer, A.C. Geurts, V. Weerdesteyn, Deficits in motor response to avoid sudden obstacles during gait in functional walkers poststroke, Neurorehabil. Neural Repair 27 (3) (2013) 230-239.

[13] J.A. Zeni, J.G. Richards, J.S. Higginson, Two simple methods for determining gait events during treadmill and overground walking using kinematic data, Gait Posture 27 (4) (2008) 710-714.

[14] H.C. Chen, J.A. Ashton-Miller, N.B. Alexander, A.B. Schultz, Age effects on strategies to avoid obstacles, Gait Posture 2 (1994) 139-146.

[15] K.O. McGraw, S.P. Wong, Forming inferences about some intraclass correlation coefficients, Psychol. Methods 1 (1) (1996) 30-46.

[16] D.V. Cicchetti, Guidelines, criteria, and rules of the thumb for evaluating normed and standardized assessment instruments in psychology, Psychol. Assess. 6 (4) (1994) 284-290.

[17] J.M. Bland, D.G. Altman, Measuring agreement in method comparison studies, Stat. Methods Med. Res. 8 (2) (1999) 135-160.

[18] E.R. Girden, ANOVA: Repeated Measures, Sage University Paper Series on Quantitative Applications in the Social Sciences, Newbury Park, 1992.

[19] D.V. Cicchetti, A.R. Feinstein, High agreement but low kappa: II. Resolving the paradoxes, J. Clin. Epidemiol. 43 (6) (1990) 551-558.

[20] R. Hernaez, Reliability and agreement studies: a guide for clinical investigators, Gut 64 (7) (2015) 1018-1027.

[21] P.J.M. Bank, M. Roerdink, C.E. Peper, Comparing the efficacy of metronome beeps and stepping stones to adjust gait: steps to follow! Exp. Brain Res. 209 (2) (2011) 159-169.

[22] C.E. Peper, M.J. de Dreu, M. Roerdink, Attuning one's steps to visual targets reduces comfortable walking speed in both young and older adults, Gait Posture 41 (3) (2015) 830-834. 
[23] Z. Potocanac, W. Hoogkamer, F.P. Carpes, M. Pijnappels, S.M. Verschueren, J. Duysens, Response inhibition during avoidance of virtual obstacles while walking, Gait Posture 39 (1) (2014) 641-644.

[24] C. Timmermans, M. Roerdink, M.W. van Ooijen, C.G. Meskers, T.W. Janssen, P.J Beek, Walking adaptability therapy after stroke: study protocol for a randomized controlled trial, Trials 17 (1) (2016) 425.

[25] V. Weerdesteyn, B. Nienhuis, J. Duysens, Advancing age progressively affects obstacle avoidance skills in the elderly, Hum. Mov. Sci. 24 (5-6) (2005) 865-880
[26] M.A. Brodie, N.H. Lovell, S.J. Redmond, S.R. Lord, Bottom-up subspace clustering suggests a paradigm shift to prevent fall injuries, Med. Hypotheses 84 (4) (2015) 356-362.

[27] C.K. Balasubramanian, The community balance and mobility scale alleviates the ceiling effects observed in the currently used gait and balance assessments for the community-dwelling older adults, J. Geriatr. Phys. Ther. 38 (2) (2015) 78-89. 